\title{
Inverse Spin-Valve-Type Magnetoresistance in Spin Engineered Multilayered Structures
}

\author{
J. M. George, L. G. Pereira, A. Barthélémy, F. Petroff, L. Steren, J. L. Duvail, and A. Fert \\ Laboratoire de Physique des Solides, Bâtiment 510, Université Paris-Sud, 91405 Orsay Cédex, France
}

R. Loloee, P. Holody, and P. A. Schroeder

Department of Physics and Astronomy and Center for Fundamental Materials Research, Michigan State Unicersity, East Lansing, Michigan 48824

(Received 28 July 1993)

\begin{abstract}
The resistivity of magnetic multilayers is generally smaller when the magnetizations of successive layers are parallel, which is the so-called giant magnetoresistance or spin-valve effect. We have been able to reverse this effect and to obtain a smaller resistivity for an antiparallel arrangement by intercalating thin $\mathrm{Cr}$ layers within half of the $\mathrm{Fe}$ layers in $\mathrm{Fe} / \mathrm{Cu}$ multilayers. This inverse spin-valve effect is due to the inverse spin asymmetries of the electron scattering in successive Fe layers with and without $\mathrm{Cr}$. This is a confirmation of the fundamental mechanism of the giant magnetoresistance.
\end{abstract}

PACS numbers: $75.50 . \mathrm{Rr}, 73.40 . \mathrm{Jn}, 75.70 . \mathrm{Cn}$

Giant magnetoresistance (GMR) effects [1,2] have now been observed in many magnetic multilayered structures. The resistivity is larger in the antiferromagnetic configuration, that is, when the magnetizations of successive magnetic layers are antiparallel. These magnetoresistance (MR) effects are currently ascribed to the conduction in parallel by the two spin directions and to the spin dependence of the electron scattering in the magnetic layers or at their interfaces $[1,3,4]$.

The schematic of Fig. 1 illustrates the mechanism in the simple limit where the electron mean free path is much larger than the layer thicknesses. For simplicity the figure is drawn with only scattering at the interfaces (but there is also scattering within the layers). The schematic is for the case of minority spin (spin $\downarrow$ ) electrons more strongly scattered than the majority spin (spin $\uparrow$ ) electron, i.e., $\alpha=\rho \downarrow / \rho \uparrow>1$ in the conventional notation [5]. When, as represented on the right, the magnetization of all the magnetic layers is parallel (high field ferromagnetic configuration), the conduction electrons with spin + (i.e., $s_{z}=+\frac{1}{2}$ ) are weakly scattered in all the layers, and form a low resistivity channel. The shunting of the current by this channel produces a low resistivity in the ferromagnetic (F) configuration. This is the normal spin-valve effect. If the two current picture is correct, it should be possible to reverse this MR effect by alternating magnetic layers $M_{1}$ and $M_{2}$ with $\alpha_{1}<1$ and $\alpha_{2}>1$, respectively, as illustrated by Fig. 1(b). In the antiferromagnetic (AF) configuration represented at the right of Fig. 1(b), the spins + are weakly scattered minority electrons in layer $M_{1}$ (since $\left.\alpha_{1}<1\right)$ and again weakly scattered minority electrons in the antiparallel layer $M_{2}$ (since now $\alpha_{2}>1$ ). The shunting effect by this channel makes the resistivity smaller in the AF configuration, in contrast with the normal situation where the shunting effect occurs in the $F$ configuration. A reversed MR is therefore expected in the situation of Fig. 1(b), with $\alpha_{1}<1$ and $\alpha_{2}>1$. Combinations of scatterings with $\alpha$ smaller and larger than 1 have already been used to
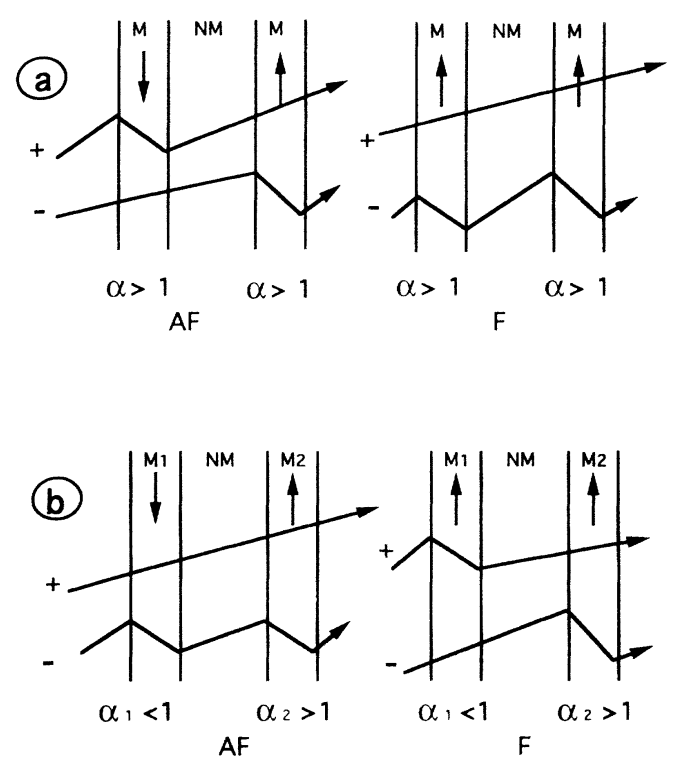

FIG. 1. Schematic picture of spin-valve effects in magnetic/nonmagnetic multilayers. Arrows indicate the majority spin direction in the magnetic layers for antiferromagnetic (AF, left) and ferromagnetic ( $F$, right) arrangements. The electron trajectories between two scatterings are represented by straight lines and the scatterings by abrupt changes in direction. The spins + and - are for electron spins $s_{z}=+\frac{1}{2}$ and $s_{z}=-\frac{1}{2}$, respectively. (a) Normal $M R$ : The spin asymmetry coefficient is the same for all the magnetic layers and is supposed to be larger than 1 for the figure. In the $F$ configuration (right), the spin + electrons $\left(s_{z}=+\frac{1}{2}\right)$ are weakly scattered by all the magnetic layers and the shunting of the current by this low resistivity channel makes the resistivity low in the $\mathrm{F}$ state, i.e., $\rho_{\mathrm{AF}}=\rho \uparrow \rho \downarrow /(\rho \uparrow+\rho \downarrow)<\rho_{\mathrm{AF}}=(\rho \uparrow+\rho \downarrow) / 4$. (b) Reversed $M R$ : The spin asymmetry coefficient is supposed to be smaller than 1 in the magnetic layer $M_{1}$ and larger than 1 in $M_{2}$. In the AF configuration (left), the spin + are weakly scattered minority spins in layer $M_{1}$ (since $\alpha_{1}<1$ ) and also weakly scattered majority spins in layer $M_{2}$ (since $\left.\alpha_{2}<1\right)$. The shunting of the current by the spin + channel now occurs in the AF configuration, i.e., $\rho_{\mathrm{AF}}<\rho_{\mathrm{F}}$ (inverse MR). 
modify the GMR [6], but to our knowledge, never to reverse it (that is, with $\alpha_{1}<1$ and $\alpha_{2}>1$ in successive layers, as described above).

We have performed MR measurements at room temperature with an usual four point technique on $\left(M_{1} / \mathrm{Cu} /\right.$ $\left.M_{2} / \mathrm{Cu}\right) \times 5$ multilayers grown by dc magnetron sputtering. The structure of the multilayers is shown in Fig. 2:

(i) The magnetic layer $M_{2}$ is a Fe layer. From electronic structure arguments $[7,8]$, its asymmetry coefficient $\alpha_{2}$ is expected to be larger than 1 .

(ii) The magnetic layer $M_{1}$ is composed of $24 \AA$ of $\mathrm{Fe}$ in the center of which we have inserted $4 \AA$ of $\mathrm{Cr}$. This thickness of $\mathrm{Cr}$ has been chosen to produce a ferromagnetic coupling between the two parts of the Fe layer [9] and to ensure that $M_{1}$ behaves as a unique magnetic layer (we will, however, see that the ferromagnetic alignment is not perfect). Chromium has been chosen because the scattering by the $\mathrm{Fe} / \mathrm{Cr}$ interfaces is known to be strongly spin dependent [7], with probably $\alpha<1$ (by analogy with the case of $\mathrm{Cr}$ impurities in $\mathrm{Fe}$ [5] and also from other electronic structure arguments [8]). Of course, the scattering with $\alpha<1$ by the $\mathrm{Fe} / \mathrm{Cr}$ interfaces is not the only one in layer $M_{1}$ and competes with scattering with $\alpha>1$ at the $\mathrm{Fe} / \mathrm{Cu}$ interfaces of $M_{1}$. Nevertheless, from the much higher $\mathrm{MR}$ in $\mathrm{Fe} / \mathrm{Cr}$ multilayers compared to $\mathrm{Fe} / \mathrm{Cu}$, one knows that the spin asymmetry at the $\mathrm{Fe} / \mathrm{Cr}$ interfaces is more pronounced than at the $\mathrm{Fe} / \mathrm{Cu}$ interfaces [i.e., $1 / \alpha(\mathrm{Fe} / \mathrm{Cr})>\alpha(\mathrm{Fe} / \mathrm{Cu})$ ], so that the global spin asymmetry coefficient $\alpha_{1}$ can be expected to be smaller than 1 .

(iii) With $\alpha_{1}<1$ and $\alpha_{2}>1$, the additional condition to obtain a reversed MR is to have an AF exchange coupling between $M_{1}$ and $M_{2}$ across a $\mathrm{Cu}$ layer. According to experimental data on $\mathrm{Fe} / \mathrm{Cu}$ multilayers prepared by sputtering in similar conditions [10], a maximum of AF coupling is obtained for a $\mathrm{Cu}$ thickness $t_{\mathrm{Cu}}$ around $14 \AA$ (first $\mathrm{AF}$ peak). In fact, for a more rigorous test, we have prepared samples with different values of $t_{\mathrm{Cu}}$ between 10 and $20 \AA$ in order to check that the inverse MR is maximum around the AF peak, disappears for ferromagnetic coupling $\left(t_{\mathrm{Cu}}=10 \AA\right)$, and is definitely reduced for weak

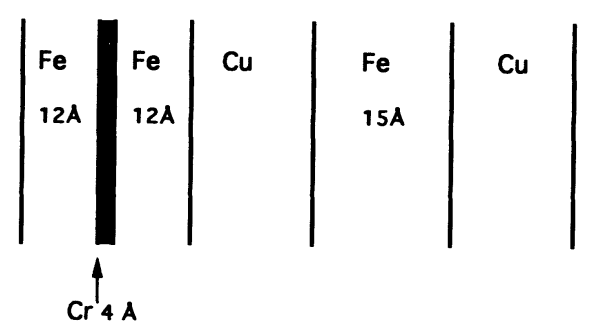

FIG. 2. Structure of the multilayers exhibiting inverse MR. One period is represented in the figure. Two iron layers of $12 \AA$ coupled ferromagnetically through a thin $\mathrm{Cr}$ layer $(4 \AA)$ compose the magnetic layer $M_{1}$. The magnetic layer $M_{2}$ is a $\mathrm{Fe}$ layer of $15 \AA$ and is separated from $M_{1}$ by a layer of $\mathrm{Cu}$. The final structure is $\left(M_{1} / \mathrm{Cu} / M_{2} / \mathrm{Cu}\right) \times 5$. ferromagnetic coupling $\left(t_{\mathrm{Cu}}=20 \AA\right)$.

As illustrated in Fig. 3, a reversed MR is observed for thicknesses of $\mathrm{Cu}$ providing an antiferromagnetic coupling, with the highest inverse effect for $t_{\mathrm{Cu}}=16 \AA$, close to the maximum of AF coupling (we will come back later to the correlated variation of the MR and AF coupling). The inverse MR is seen independently of the direction of the field; i.e., the MR curves are very similar for fields parallel and perpendicular to the current in the layer plane. This demonstrates that the MR we observe cannot be due to the so-called anisotropic magnetoresistance (or AMR, resulting from the spontaneous resistivity anisotropy of ferromagnets [5]), and can only be an inverse spinvalve effect. As a matter of fact, the maximum AMR amplitude, measured by rotating the field at saturation, does not exceed $0.2 \%$ in our samples, an order of magnitude below the MR effects in Fig. 3.

By looking at the magnetization curve for the sample with $t_{\mathrm{Cu}}=16 \AA$ in Fig. 3 , we first note that the remanent magnetization is only $14 \%$ of the magnetization at saturation, which is typical of antiferromagnetic coupling in multilayers. We also note the usual correlation between the field dependences of the resistivity and magnetization. In particular, both the reversed magnetoresistance and magnetization are saturated at about the same relatively low field, around $150 \mathrm{G}$. This low field, typical of the relatively weak AF coupling in $\mathrm{Fe} / \mathrm{Cu}[10]$, is the field

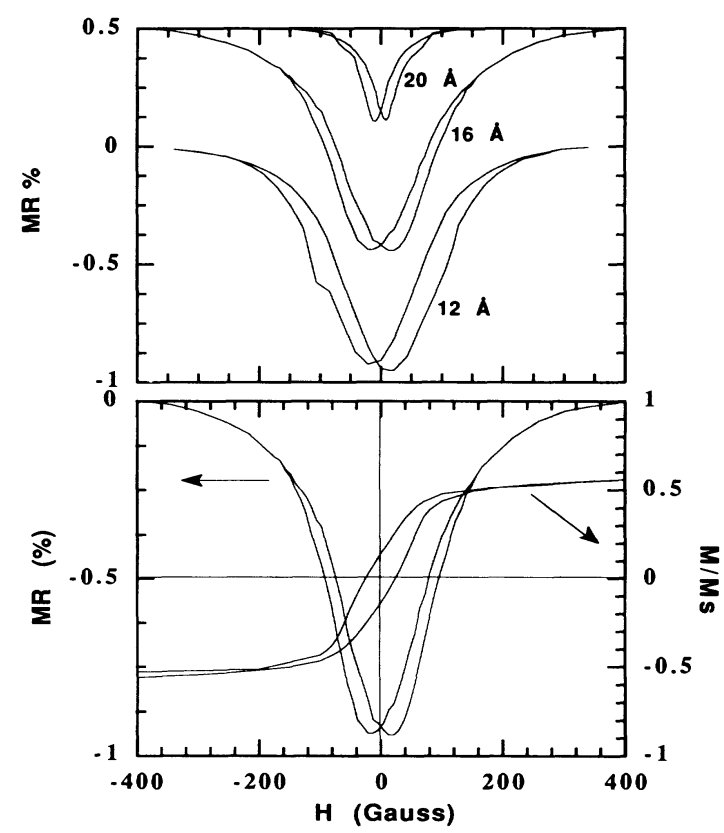

FIG. 3. Low field MR curves for several ([Fe $12 \AA / \mathrm{Cr} 4$ $\left.\AA / \mathrm{Fe} 12 \AA] / \mathrm{Cu} t_{\mathrm{Cu}} / \mathrm{Fe} 15 \AA / \mathrm{Cu} t_{\mathrm{Cu}}\right) \times 5$ samples are represented in the upper figure. The thickness of $\mathrm{Cu}, t_{\mathrm{Cu}}$, is indicated on the curves. For clarity, the curves for $t_{\mathrm{Cu}}=16$ and $20 \AA$ are shifted upward. For the sample with $t \mathrm{Cu}=16 \AA$, the low field magnetization curve is also shown and can be compared to the MR (lower figure). 
which aligns the magnetic moments of the $\mathrm{FeCrFe}$ and Fe layers on both sides of $\mathrm{Cu}$.

By looking more carefully at the magnetization data in Fig. 3, we also notice that the saturation at about $150 \mathrm{G}$ is far from being complete; above $150 \mathrm{G}$, the magnetization continues to increase very slowly and almost linearly. Proceeding to Fig. 4 which represents the magnetization loop in a much larger field range, we see that the magnetization continues to increase slowly and is saturated only at a much higher field around $10^{4} \mathrm{G}$. This slow increase at high field that we observe in the $\mathrm{FeCrFe} / \mathrm{Cu} / \mathrm{Fe} / \mathrm{Cu}$ samples as well as in simple $\mathrm{FeCrFe}$ layers can be ascribed to an imperfect ferromagnetic coupling across $\mathrm{Cr}$. If, for example, we imagine some mixing of AF and $\mathrm{F}$ coupling inducing a tilt [11] between the magnetic moments on both sides of $\mathrm{Cr}$, a straightforward calculation shows that an average tilt of $59^{\circ}$ corresponds to the intermediate saturation step at about $70 \%$ (this value of $M / M_{s}$ is derived by a linear extrapolation to zero field in Fig. 4). We thus have a saturation in two stages: A small field of about $150 \mathrm{G}$ is sufficient to align the magnetic moments of the $\mathrm{FeCrFe}$ and Fe layers, and a field exceeding $10^{4} \mathrm{G}$ is necessary to obtain a perfect ferromagnetic alignment within the $\mathrm{FeCrFe}$ layers.

The improvement of the ferromagnetic alignment of the Fe half layers on both sides of $\mathrm{Cr}$ in the $\mathrm{FeCrFe}$ layers is normally expected to induce a normal spin-valve MR at high field, in addition to the reversed MR associated to the relative orientation of the magnetic moments of the $\mathrm{Fe}$ and $\mathrm{FeCrFe}$ layers on both sides of $\mathrm{Cu}$. This is

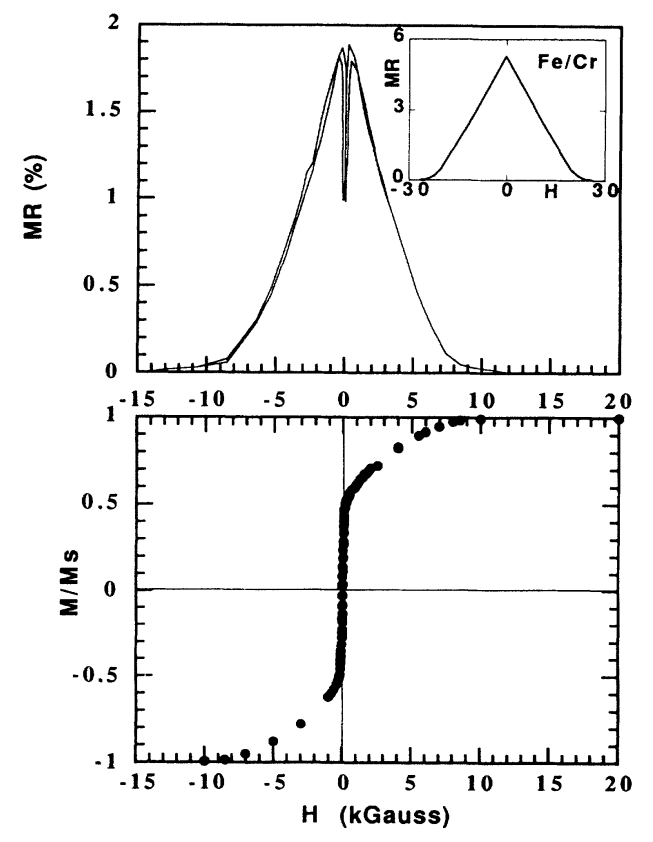

FIG. 4. High field MR and magnetization curves for ([Fe 12 $\AA / \mathrm{Cr} 4 \AA / \mathrm{Fe} 12 \AA] / \mathrm{Cu} 16 \AA / \mathrm{Fe} 15 \AA / \mathrm{Cu} 16 \AA) \times 5$. Inset: MR curve for $(\mathrm{Fe} 12 \AA / \mathrm{Cr} 6 \AA) \times 10$. what we observe when the MR is recorded in a very large field range; see Fig. 4. As shown in the inset of Fig. 4, a similar high field behavior is also found in simple $\mathrm{Fe} / \mathrm{Cr} 6$ $\AA$ multilayers. For $\mathrm{FeCrFe} / \mathrm{Cu} / \mathrm{Fe} / \mathrm{Cu}$ samples, the low field inverse MR adds to the high field normal MR only present in $\mathrm{Fe} / \mathrm{Cr}$. We must admit that this mixing of low field inverse and high field normal MR makes the inverse MR effect less clear than might have been expected. However, we emphasize that the inverse and normal MR are observed in clearly different field ranges: The inverse MR is clearly related to the relative orientation of the magnetic moments of the $\mathrm{Fe}$ and $\mathrm{FeCrFe}$ layers, while the normal MR appears in the high field range characteristic of the coupling across $4 \AA$ of $\mathrm{Cr}$. In addition, to reinforce our interpretation, we have performed several experimental tests that we describe below.

First, as we pointed out above, a reversed MR is expected for $\alpha_{1}<1$ and $\alpha_{2}>1$ (or vice versa) and should disappear for $\alpha_{1}=\alpha_{2}$, i.e., $M_{1}=M_{2}$. For $M_{1}=M_{2}=\mathrm{Fe}$, that is, for $\mathrm{Fe} / \mathrm{Cu}$, one already knows that the spin-valve MR is normal (nonreversed) [10]. We also looked at the case $M_{1}=M_{2}=\mathrm{FeCrFe}$ with samples of the type Fe $12 \AA$ $\mathrm{Cr} 4 \AA \mathrm{Fe} 12 \AA / \mathrm{Cu} 14 \AA / \mathrm{Fe} 12 \AA \mathrm{Cr} 4 \AA \mathrm{Fe} 12 \AA / \mathrm{Cu} 14$ $\AA$; they present a normal (nonreversed) spin-valve $\mathrm{MR}$ at low field.

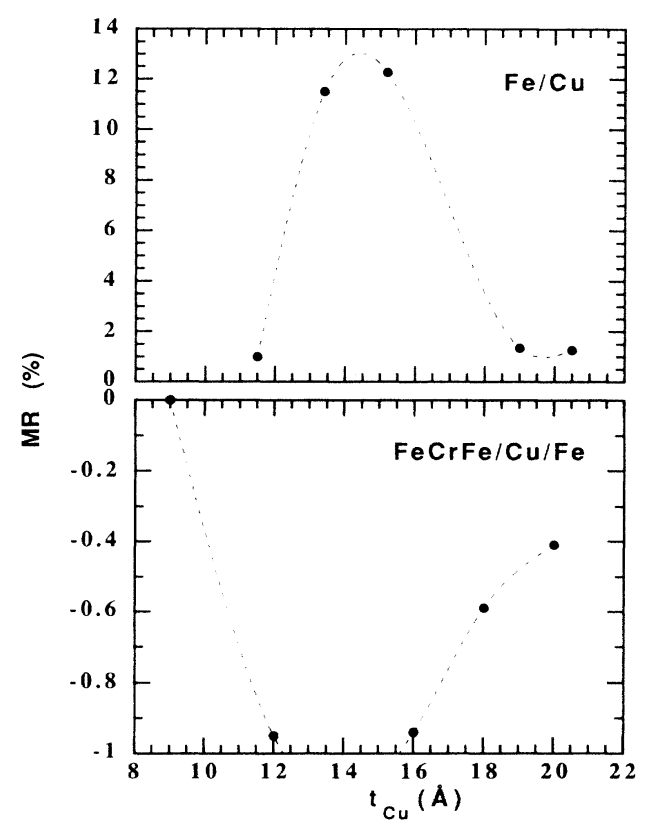

FIG. 5. Thickness dependence of the MR ratio for the normal magnetoresistance of $\mathrm{Fe} / \mathrm{Cu}$ multilayers (4.2 K, Ref. [10], upper part of the figure) and the inverse MR of the multilayered structures described in this paper (lower part of the figure). The MR ratio of the normal $M R$ is defined as $[\rho$ (low field maximum) $-\rho$ (high field) $] / \rho$ (high field). The $M R$ ratio of the inverse MR is defined as [ $\rho$ (low field minimum) $-\rho$ (maximum) $] / \rho$ (low field minimum) and, with this definition, is negative. The dashed lines are only guides for the eye. 
Second, we have to check that our inverse MR cannot be due to a second type of mechanism which, applied to our $\mathrm{FeCrFe} / \mathrm{Cu} / \mathrm{Fe} / \mathrm{Cu}$ system, can be described in the following way. Suppose some tilt between the magnetic moments of the two parts of the $\mathrm{FeCrFe}$ layer. At zero field, a $\mathrm{F}$ coupling across $\mathrm{Cu}$ will align the magnetizations on both sides of $\mathrm{Cu}$; but a moderate applied field will align the total magnetic moment of the $\mathrm{FeCrFe}$ layer with that of the Fe layer, thus disaligning the magnetizations on both sides of $\mathrm{Cu}$ and inducing a reversed MR effect. This second type of mechanism, already seen in some systems at IBM Almaden [12], necessarily implies a ferromagnetic coupling across $\mathrm{Cu}$. We have already seen that the maximum of reversed MR for $16 \AA$ of $\mathrm{Cu}$ and the corresponding very small remanent magnetization are consistent with AF coupling. More precisely, we can see in Fig. 5 the correlation between the thickness dependence of the normal $\mathrm{MR}$ in $\mathrm{Fe} / \mathrm{Cu}$ and the reversed $\mathrm{MR}$ in $\mathrm{FeCrFe} / \mathrm{Cu} / \mathrm{Fe} / \mathrm{Cu}$. Not only the inverse $\mathrm{MR}$ is $\max -$ imum where the normal $\mathrm{MR}$ in $\mathrm{Fe} / \mathrm{Cu}$ is maximum (maximum AF coupling), but also the inverse MR disappears at $t_{\mathrm{Cu}}=9 \AA$ (ferromagnetic coupling and zero MR in $\mathrm{Fe} / \mathrm{Cu}$ ) and is definitely reduced at $t_{\mathrm{Cu}}=20 \AA$ (weak $\mathrm{F}$ coupling and small $\mathrm{MR}$ in $\mathrm{Fe} / \mathrm{Cu}$ ). This convincingly shows that the inverse MR of our samples is associated to AF coupling across $\mathrm{Cu}$ and rules out the second mechanism.

We have therefore found an inverse MR of the spinvalve type in $\mathrm{Fe} / \mathrm{Cu}$ multilayers doped with thin $\mathrm{Cr}$ layers and we have shown that this change of sign can be due only to the existence of different spin asymmetry coefficients in neighbor magnetic layers, $\alpha_{1}<1$ for the $\mathrm{FeCrFe}$ layers and $\alpha_{2}>1$ for the Fe layers. The amplitude of the reversed $\mathrm{MR}$ is relatively small around $1 \%$ for $t_{\mathrm{Cu}}=16$ $\AA$, whereas a calculation in the Camley-Barnas free electron model [3] with parameters derived for $\mathrm{Fe} / \mathrm{Cu}$ and $\mathrm{FeCrFe} / \mathrm{Cu}$ multilayers predicts a few percent [13]. However, this is not completely surprising, since recent nonfree electron models have shown that channeling effects by the periodic potential of the multilayers can considerably change the magnitude of the MR predicted by free electron models $[14,15]$. The observation of inverse $M R$ is mainly interesting as a fundamental test for the basic idea of the standard interpretation of the GMR (illustrated by Fig. 1). A failure of this test would have questioned the basis of almost all the existing models. Changing the sign of the MR by introducing thin $\mathrm{Cr}$ layers is also a good example of the potential of spin engineering for modeling the properties of multilayers.

This work has been in part funded by NATO Grant No. 5-2-05/RG 890 599, the NSF under Grant No. DMR 9122614, the U.S.-France (NSF-CNRS) Cooperative Program, the Michigan State University Center for Fundamental Material Science, and Ford Scientific
Laboratory.

[1] M. N. Baibich, J. M. Broto, A. Fert, F. Nguyen Van Dau, F. Petroff, P. Etienne, A. Friederich, and J. Chazelas, Phys. Rev. Lett. 61, 2472 (1988).

[2] G. Binash, P. Grünberg, F. Saurenbach, and W. Zinn, Phys. Rev. B 39, 4828 (1989).

[3] P. E. Camley and J. Barnas, Phys. Rev. Lett. 63, 664 (1989).

[4] P. M. Levy, S. Zhang, and A. Fert, Phys. Rev. Lett. 65, 1643 (1990).

[5] A. Fert and I. A. Campbell, J. Phys. F 6, 849 (1976); I. A. Campbell and A. Fert, in Ferromagnetic Materials, edited by E. P. Wohlfarth (North-Holland, Amsterdam, 1982), Vol. 3, p. 769.

[6] P. Baumgart, B. A. Gurney, D. R. Wilhoit, B. Dieny, and V. S. Speriosu, J. Appl. Phys. 69, 4792 (1991); B. A. Gurney, P. Baumgart, B. Dieny, and V. S. Speriosu, J. Appl. Phys. 70, 5867 (1991).

[7] A. M. Gijs and M. Okada, Phys. Rev. B 46, 2908 (1992).

[8] J. Inoue and S. Maekawa, Prog. Theor. Phys. 106, 187 (1991).

[9] J. Grünberg, R. Schreiber, Y. Pang, M. B. Brodsky, and H. Sowers, Phys. Rev. Lett. 57, 2442 (1986); S. S. Parkin, N. More, and K. P. Roche, Phys. Rev. Lett. 64, 2304 (1990).

[10] F. Petroff, A. Barthélémy, D. H. Mosca, D. K. Lottis, A. Fert, P. A. Schroeder, W. P. Pratt, R. Loloee, and S. Lequien, Phys. Rev. B 44, 5355 (1991); S. Pizzini, F. Baudelet, D. Chandesris, A. Fontaine, H. Magnan, J. M. George, F. Petroff, A. Barthélémy, A. Fert, R. Loloee, and P. A. Schroeder, Phys. Rev. B 46, 1253 (1992).

[11] T. C. Slonczewski, Phys. Rev. Lett. 67, 3172 (1991).

[12] S. S. P. Parkin and V. Speriosu (private communication).

[13] The parameters of a Camley-Barnas model [3] for our multilayers are, for each spin direction, the transmission coefficient of the $\mathrm{Fe} / \mathrm{Cr}$ interfaces (involved in layer $M_{1}$ only), the transmission coefficient of the $\mathrm{Fe} / \mathrm{Cu}$ interfaces, the mean free path in $\mathrm{Fe}$ (involved in both $M_{1}$ and $M_{2}$ layers), and, finally, the mean free path in $\mathrm{Cu}$. We have determined the parameters of the $\mathrm{Fe} / \mathrm{Cu}$ interfaces and $\mathrm{Fe}$ and $\mathrm{Cu}$ layers also by fitting experimental data on $\mathrm{M}_{2} / \mathrm{Cu}$ (i.e., $\mathrm{Fe} / \mathrm{Cu}$ ) multilayers. The transmission coefficient at the $\mathrm{Fe} / \mathrm{Cr}$ interfaces is found from experimental data on $M_{1} / \mathrm{Cu}$ multilayers. Then all these parameters are combined to calculate the $M R$ of $\left(M_{1} /\right.$ $\mathrm{Cu} / \mathrm{M}_{2} / \mathrm{Cu}$ ). This calculation and tables of parameters will be presented in a longer article. As indicated in the text, this calculation leads to inverse MR of a few percent (instead of $1 \%$ or less). Channeling effects (as those described in Refs. [14,15] and not taken into account in the Camley-Barnas model) can account for this discrepancy. Also, owing to the competition between the $\mathrm{Fe} / \mathrm{Cr}$ and $\mathrm{Fe} / \mathrm{Cu}$ interfaces in layers $M_{1}$, the final result is very sensitive to any uncertainty on the parameters.

[14] R. Q. Hoods and L. M. Falicov, Phys. Rev. B 46, 8287 (1992).

[15] S. Zhang and P. M. Levy, Mater. Res. Soc. Symp. Proc. 313, 53 (1993). 


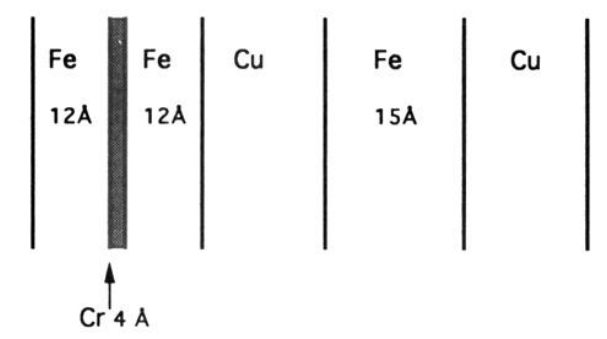

FIG. 2. Structure of the multilayers exhibiting inverse MR. One period is represented in the figure. Two iron layers of $12 \AA$ coupled ferromagnetically through a thin $\mathrm{Cr}$ layer $(4 \AA)$ compose the magnetic layer $M_{1}$. The magnetic layer $M_{2}$ is a $\mathrm{Fe}$ layer of $15 \AA$ and is separated from $M_{1}$ by a layer of $\mathrm{Cu}$. The final structure is $\left(M_{1} / \mathrm{Cu} / M_{2} / \mathrm{Cu}\right) \times 5$. 